\title{
Relação entre os níveis de vitamina A e os marcadores bioquímicos do estado nutricional de ferro em crianças e adolescentes
}

\author{
Relationship between vitamin A and biochemical \\ markers of iron status in children and adolescents
}

Rita de Cássia Ribeiro SILVA ${ }^{1}$

Ana Marlúcia Oliveira ASSIS ${ }^{1}$

Mônica Leila Portela de SANTANA ${ }^{1}$

Maurício Lima BARRETO

Luciara Leite BRITO²

Mitermayer Galvão REIS 3

Isabel Martin PARRAGA 4

Ronald Edward BLANTON ${ }^{5}$

R E S U M O

\section{Objetivo}

Verificar a relação entre vitamina $A$ e os marcadores bioquímicos da situação nutricional do ferro.

\section{Métodos}

Participaram deste estudo 178 indivíduos com idade entre 7 e 17 anos, residentes na cidade de Jequié (BA). Os indivíduos foram submetidos a exame de sangue para dosagem de retinol e de marcadores bioquímicos do estado nutricional relativo ao ferro. Além disso, foram realizados exames antropométricos, parasitológico de fezes e coletadas informações de consumo dietético de ferro e das condições sócio-ambiental e domiciliar da família. Utilizou-se a análise de regressão linear múltipla como técnica estatística para avaliar a associação de interesse.

\section{Resultados}

Constatou-se associação positiva e estatisticamente significante entre os níveis de retinol sérico e a concentração de hemoglobina $(p=0,007)$, ferro sérico $(p=0,010)$ e transferrina saturada $(p=0,027)$. Esses efeitos se mantiveram após ajuste dos modelos pelas variáveis demográficas, infecções parasitárias (Schistosoma mansoni, Trichiura

\footnotetext{
${ }^{1}$ Universidade Federal da Bahia, Escola de Nutrição. R. Araújo Pinho, 32, Campus Universitário Canela, 40110-150, Salvador,

BA, Brasil. Correspondência para/Correspondence to: R.C.R. SILVA. E-mail: <rcrsilva@ufba.br>.

2 Universidade Federal da Bahia, Instituto de Saúde Coletiva. Salvador, BA, Brasil.

3 Fundação Oswaldo Cruz, Centro de Pesquisa Gonçalo Muniz. Rio de Janeiro, RJ, Brasil.

${ }^{4}$ Case Western Reserve University, Department of Nutrition. Cleveland, Ohio, United States

${ }^{5}$ Case Western Reserve University, Center for International Health and Disease. Cleveland, Ohio, United States.
} 
286 | R.C.R. SILVA et al.

trichuris, Ascaris lumbricoides e ancilostomídeos), consumo de ferro alimentar biodisponível e condições ambientais e domiciliares.

\section{Conclusão}

A vitamina A parece contribuir para elevar o ferro orgânico da população, em especial, em áreas onde a deficiência de vitamina A e a anemia coexistem.

Termos de indexação: Adolescente. Criança. Deficiência de ferro. Deficiência de vitamina A.

\section{A B S T R A C T}

\section{Objective}

The objective of this study was to verify the relationship between vitamin A and the biochemical markers of iron status.

\section{Methods}

A total of 178 individuals aging from 7 to 17 years participated in this study, all living in Jequié, BA. The individuals were submitted to blood tests to dose retinol and biochemical iron markers. Furthermore, anthropometric measurements and stool analyses were done and dietary information regarding iron intake, socio-environmental and home conditions of the family were collected. Multiple linear regression analysis was done to assess the association of interest.

\section{Results}

A positive and statistically significant association was found between the levels of serum retinol and hemoglobin $(p=0.007)$, serum iron $(p=0.010)$ and iron-saturated tranferrin $(p=0.027)$. These findings remained unchanged after adjusting the models for demographic variables, parasitic infections (Schistosoma mansoni, Trichiura trichuris, Ascaris lumbricoides and ancylostomiasis), consumption of bioavailable dietary iron and environmental and home conditions.

\section{Conclusion}

Vitamin A seems to contribute to the increase of organic iron of the population, especially in areas were deficiency of vitamin A and iron-deficiency anemia coexist.

Indexing terms: Adolescent. Child. Iron deficiency. Vitamin A deficiency.

\section{N T R O D U ÇÃ O}

A relação entre hipovitaminose $A$ e deficiência de ferro tem sido investigada em humanos e animais experimentais e os resultados sugerem que a deficiência de vitamina $A$ na dieta pode contribuir com a redução dos níveis de ferro no organismo ${ }^{1}$. Argumenta-se que a deficiência de vitamina A compromete a mobilização de ferro armazenado, resultando em concentrações insuficientes para a medula óssea, de maneira a prejudicar a hematopoiese $e^{2-5}$.

Nesse sentido, alguns estudos têm mostrado que a suplementação com vitamina A eleva os níveis de ferro em mulheres durante a gestação ${ }^{6,7}$, em crianças jovens ${ }^{8-10}$ e em adolescentes $^{11}$, independentemente do fornecimento de ferro. A suplementação dietética com vitamina A oferecida a crianças menores de 9 anos na Tailândia, por curto período de tempo (2 semanas) e por período mais longo (4 meses), aumentou os estoques de ferro e a resistência às infecções ${ }^{9,10,12}$.

Aprofundar o conhecimento sobre a relação entre deficiência de vitamina A e a diminuição da concentração dos níveis de ferro é pertinente não somente na perspectiva científica, mas devido às suas implicações para as políticas nutricionais. Na atualidade, a política adotada pelo Ministério da Saúde de suplementação periódica de altas doses de vitamina A para as crianças de regiões onde a deficiência clínica ou sub-clínica da vitamina é endêmica, poderá também favorecer o combate à anemia ferropriva. Diante do exposto, este trabalho tem como objetivo estudar a relação 
entre retinol sérico e os marcadores bioquímicos da situação nutricional do ferro em escolares residentes em um município do Sudoeste baiano.

\section{MÉ TO D OS}

Fizeram parte deste estudo, 187 escolares de 7 a 17 anos de idade, residentes na cidade de Jequié (BA), município com 120396 habitantes. Esta cidade está situada em um dos mais prósperos municípios do Estado, possui um centro comercial e de serviços diversificados, constituindo-se em um pólo econômico de atração regional para as cidades circunvizinhas e áreas rurais. A amostra deste estudo é oriunda de uma investigação mais ampla, planejada para avaliar os fatores de risco para anemia ferropriva em crianças e adolescentes parasitados por helmintos intestinais ${ }^{12}$. Dos 1706 sujeitos selecionados para o referido estudo, foram aleatoriamente amostrados 128 escolares. Esse número foi estimado com base no poder de $80 \%$ (1- $\beta$ ) de detectar uma prevalência de $30 \%$ de anemia nesse grupo etário ${ }^{12}$. Foi acrescido à amostra um percentual ao redor de $50 \%$, prevendo possíveis perdas na coleta de sangue e a estratificação de variáveis na análise de dados. Dada a perda de 13 indivíduos no processo de coleta de sangue, integraram a amostra final do presente estudo 187 escolares. Este número de escolares amostrado tem um poder de $82 \%$ (1- $\beta$ ) para detectar uma prevalência de 30\% da anemia, com um nível de significância de 0,05 (1- $\alpha$ ).

Para avaliar o estado nutricional relativo à vitamina A e ao ferro foram adotados os indicadores e seus respectivos pontos de corte, tradicionalmente usados em estudos epidemiológicos, conforme recomendado pela United Nations Children's Fundation (UNICEF) ${ }^{13}$.

O retinol sérico foi determinado por meio do Cromatografia Liquida de Alta Performance (HPLC), de acordo com o método de Bieri et al. ${ }^{14}$. Para a determinação do ferro sérico, foi utilizado o "kit" in vitro Diagnóstica CAT 015, que adota o método Goodwim modificado (Ferrozine). O cálculo da saturação da transferrina foi realizado pelo método indireto, a partir da razão entre ferro sérico e capacidade total de ligação de ferro multiplicada por 100. Os níveis de concentração de hemoglobina foram medidos por meio do hemoglobinômetro portátil (HEMOCUE, Inc., Laguna Hills, CA $)^{15}$.

A identificação de S. mansoni e geohelmintos foi realizada por meio da técnica quantitativa de Kato-Katz ${ }^{16}$. O exame para a identificação dos ovos de ancilostomídeos foi realizado duas horas após a preparação das lâminas. A estimativa do número de ovos eliminado por grama de fezes do S. mansoni e geohelmintos, foi obtida a partir do número de ovos encontrado em cada lâmina de Kato, multiplicado pela constante 24 .

Foram obtidos ainda dados sobre saneamento ambiental (abastecimento de água, esgoto e coleta pública de lixo), e condições de moradia (tipo de construção, número de pessoas por cômodo, instalação sanitária). Para coleta desses dados utilizou-se um questionário padronizado, aplicado aos chefes de família ou aos responsáveis pelos escolares. Essas variáveis deram origem ao indicador das condições sócio-ambiental e domiciliar. Fizeram parte desse indicador todas as variáveis que permaneceram significantemente associadas à anemia $(p<0,05)$ na análise multivariada: origem da água, freqüência de abastecimento de água, lugar onde é guardada a água, cuidado com água de beber, existência de vaso sanitário, destino dos dejetos, freqüência de coleta de lixo, existência de terreno baldio com lixo, existência de esgoto a céu aberto, tipo de piso, tipo de domicílio, tipo de pavimentação e tipo de parede. $\mathrm{O}$ indicador foi totalizado e classificado em estrato adequado (escore ambiental de 0 a 6) e estrato inadequado (escore de 7 a 13). O detalhamento da construção desse indicador encontra-se publicado por Brito et al. ${ }^{12}$.

O inquérito recordatório de 24 horas foi o método utilizado para definir o consumo alimentar da população. Para facilitar o processo recordatório do informante, um álbum contendo desenhos dos alimentos com porcionamentos diferenciados foi utilizado para identificar o tamanho das porções, assegurando, dessa maneira, a melhoria da qualidade da informação. Para o cálculo de energia, 
macro e micronutrientes foi utilizado o programa de cálculo da composição centesimal da dieta Virtual Nutri - USP ${ }^{17}$. O ferro biodisponível foi quantificado pela equação desenvolvida por Monsen \& Balintfy ${ }^{18}$.

A normalidade da distribuição dos dados foi verificada por meio do teste KolmogorovSmirnov. Os dados foram logaritimicamente normalizados, quando necessário. Utilizou-se a análise de regressão linear multivariada para avaliar as associações de interesse. Os marcadores bioquímicos do estado nutricional relativo ao ferro - hemoglobina, ferro sérico, capacidade total de ligação do ferro e saturação da transferrina (\%) - constituíram as variáveis dependentes em cada um dos modelos apresentados. A variável independente foi representada pelo nível de retinol sérico. Dados demográficas, sócio-ambientais e domiciliares, consumo de ferro biodisponível e carga parasitária de infecção pelo S. mansoni e geohelmintos (em log $(x+1))$ foram adotados como variáveis de ajuste. Foram utilizados testes bicaudais e nível de significância de 5\%. Os dados foram analisados com o pacote estatístico Statistical Package for the Social Sciences (SPSS/PC)+ (versão 10,0).

O protocolo do estudo foi submetido à Comissão de Ética da Fundação Oswaldo Cruz, que apreciou e emitiu parecer favorável sobre a pertinência ética da investigação. Após a informação detalhada dos riscos e procedimentos a que seus filhos seriam submetidos na pesquisa, foi solicitado aos pais ou responsáveis por cada criança um consentimento escrito, e somente aquelas cujos pais aceitaram em participar foram incluídas no estudo. Todos os sujeitos arrolados neste estudo tiveram o termo de consentimento livre e esclarecido para a participação assinado pelos pais ou responsáveis.

\section{RES U LTA D O S}

O grupo estudado, como demonstrado na Tabela 1, se caracterizou pelo discreto predomínio do sexo masculino (51,9\%); com idade entre 10 e 13 anos de idade (53,5\%); renda mensal entre 1 e 3 salários mínimos (51,9\%); ausência de formação escolar dos respectivos chefes de famílias (48,6\% eram analfabetos) e moradia em condições ambientais e domiciliares inadequadas $(52,0 \%)$.

Constatou-se, a partir dos dados da Tabela 2, associação positiva e estatisticamente significante, entre níveis de retinol sérico e a concentração de hemoglobina $(p=0,007)$, ferro sérico $(p=0,010)$ e saturação da transferrina $(p=0,027)$. Esses efeitos se mantiveram mesmo após ajuste das variáveis demográficas, infecção parasitárias (Schistosoma mansoni, A. lumbricoides T. trichuris e ancilostomídeo), consumo de ferro biodisponível e condições ambientais e domiciliares nos diversos modelos apresentados. Em cada um desses modelos identificou-se elevação de $0,033 \mathrm{~g} / \mathrm{dL}$, $0,824 \mathrm{mg} / \mathrm{dL}$, e 0,266\%, respectivamente, na concentração de hemoglobina, ferro sérico e saturação da transferrina, a cada unidade de aumento de retinol sérico ( $\mu \mathrm{mol} / \mathrm{L})$.

Na Tabela 3 estão listados os alimentos mais freqüentes nas principais refeições realizadas pelos participantes. De acordo com os dados, as refeições que mais contribuíram para o fornecimento de ferro foram o almoço e o jantar. Ao

Tabela 1. Caracterização demográfica e socioeconômica de escolares. Jequié (BA), 1997-1998.

\begin{tabular}{lcc}
\hline Variáveis & $\mathrm{n}$ & $\%$ \\
\hline Sexo & 90 & 48,1 \\
Feminino & 97 & 51,9 \\
Masculino & & \\
Idade & 22 & 11,8 \\
7-9 anos & 100 & 53,5 \\
10-13 anos & 65 & 34,8 \\
14-19 anos & & \\
Escolaridade do chefe & 90 & 48,6 \\
Analfabeto & 79 & 42,7 \\
Primário incompleto/completo & 16 & 8,6 \\
Ginásio incompleto/completo/superior & & \\
Renda familiar & 71 & 38,0 \\
<1 salário-mínimo (SM) & 97 & 51,9 \\
1SM - 3SM & 19 & 10,2 \\
>3SM & & \\
Condições sócio-ambientais e domiciliares & 86 & 52,0 \\
Inadequadas & 93 & 48,0 \\
Adequadas & &
\end{tabular}

SM: salário mínimo. 
Tabela 2. Associação entre marcadores bioquímicos do estado nutricional relativo ao ferro e vitamina $A$ em escolares. Jequié (BA), 1997-1998.

\begin{tabular}{|c|c|c|c|}
\hline Variável dependente & Beta & Erro-padrão & $P$ valor \\
\hline \multicolumn{4}{|c|}{ Modelo I *- Hemoglobina (g/dL) } \\
\hline \multicolumn{4}{|c|}{ Constante $-10,094$} \\
\hline Vitamina A $(\mu \mathrm{mol} / \mathrm{L})$ & 0,033 & 0,012 & 0,007 \\
\hline \multicolumn{4}{|c|}{ Modelo II* - Ferro Sérico (mg/dL) } \\
\hline \multicolumn{4}{|c|}{ Constante - 34,446 } \\
\hline Vitamina A $(\mu \mathrm{mol} / \mathrm{L})$ & 0,824 & 0,315 & 0,010 \\
\hline \multicolumn{4}{|c|}{ Modelo III* - Capacidade total ligadora do ferro $(\mu \mathrm{g} / \mathrm{dL})$} \\
\hline \multicolumn{4}{|c|}{ Constante - 315,84 } \\
\hline Vitamina A ( $\mu \mathrm{mol} / \mathrm{L})$ & 1,448 & 0,736 & 0,052 \\
\hline \multicolumn{4}{|c|}{ Modelo IV* - Saturação da Transferrina (\%) } \\
\hline \multicolumn{4}{|c|}{ Constante $-8,39$} \\
\hline Vitamina A ( $\mu \mathrm{mol} / \mathrm{L})$ & 0,266 & 0,097 & 0,027 \\
\hline
\end{tabular}

Coeficiente de regressão linear múltipla com hemoglobina, ferro sérico, capacidade total ligadora de ferro (CTLF) e transferrina saturada como variáveis dependentes e retinol sérico $(\mu \mathrm{mo} / \mathrm{l} / \mathrm{)}$ como variável independente; *Coeficientes ajustados por idade (em anos), sexo, ferro biodisponível, Infecção pelo S.mansoni, A. lumbricoides, T Trichuris, ancilostomídeo e pelo indicador das condições sócio-ambientais e domiciliares.

Tabela 3. Contribuição dos diferentes alimentos no fornecimento de ferro dietético a escolares, de acordo com as refeições realizadas. Jequié (BA), 1997-1998.

\begin{tabular}{|c|c|c|c|c|}
\hline Refeição & Alimento & Porção média & $* * \mathrm{mg}^{\mathrm{a}}$ & $\% * * *$ \\
\hline \multicolumn{5}{|l|}{ Desjejum } \\
\hline & Café & $178,3 \mathrm{~mL}$ & $0,7 \quad(0,6)$ & 80,4 \\
\hline & Raízes e tubérculos & $159,9 \mathrm{gL}$ & $0,9 \quad(0,7)$ & 7,9 \\
\hline & Frutasc & $149,3 \mathrm{gL}$ & $1,0 \quad(1,0)$ & 2,4 \\
\hline & Pão/biscoito & $77,4 \mathrm{gL}$ & $0,3 \quad(1,0)$ & 90,4 \\
\hline & Leite in natura & $139,3 \mathrm{~mL}$ & $0,2 \quad(0,1)$ & 15,6 \\
\hline \multicolumn{5}{|l|}{ Almoço } \\
\hline & Fígado & $31,80 \mathrm{~g}$ & $1,5 \quad(1,4)$ & 1,2 \\
\hline & Carne de boi & $36,55 \mathrm{~g}$ & $0,9 \quad(1,8)$ & 69,8 \\
\hline & Frango & $44,60 \mathrm{~g}$ & $0,6 \quad(0,6)$ & 15,8 \\
\hline & Ovo de galinha & $58,10 \mathrm{~g}$ & $1,3 \quad(1,4)$ & 4,2 \\
\hline & Feijão & $171,90 \mathrm{~g}$ & $2,6 \quad(2,4)$ & 90,2 \\
\hline & Verduras $^{d}$ & $32,90 \mathrm{~g}$ & $0,2 \quad(0,3)$ & 22,9 \\
\hline & Farinha de mandioca & $83,30 \mathrm{~g}$ & $0,8 \quad(0,6)$ & 39,7 \\
\hline & Frutas ${ }^{c}$ & $95,40 \mathrm{~g}$ & $0,3 \quad(0,5)$ & 10,4 \\
\hline \multicolumn{5}{|l|}{ Jantar } \\
\hline & Carne de boi & $36,10 \mathrm{~g}$ & $0,7 \quad(1,1)$ & 41,7 \\
\hline & Frango & $22,00 \mathrm{~g}$ & $0,8 \quad(0,5)$ & 8,2 \\
\hline & Feijão & $161,20 \mathrm{~g}$ & $2,4 \quad(2,4)$ & 42,3 \\
\hline & Frutas ${ }^{c}$ & $134,80 \mathrm{~g}$ & $0,8 \quad(1,8)$ & 2,9 \\
\hline & Leite & $148,8 \mathrm{~mL}$ & $0,2 \quad(0,1)$ & 5,8 \\
\hline & Pão & $83,90 \mathrm{~g}$ & $0,5 \quad(1,3)$ & 33,1 \\
\hline
\end{tabular}

**Porcentagem de indivíduos que consumiram os alimentos; ***Quantidade média de ferro fornecida; ${ }^{a}$ Entre parênteses desvio-padrão; bBanana da terra, batata-doce e aipim; 'Maracujá/laranja/mamão e banana; ${ }^{\mathrm{d} A l f a c e / c o u v e / t o m a t e / p e p i n o . ~}$ analisar a contribuição dos alimentos que são fontes de ferro ou favorecem sua absorção, por refeição, verificou-se uma maior contribuição para a carne de boi, seguida pelo frango, com quantidades médias ingeridas menores do que $40 \mathrm{~g}$, no caso da carne de boi, e de $45 \mathrm{~g}$, no caso do frango. O feijão contribuiu, em média, com 2,6 e $2,4 \mathrm{mg}$ de ferro no almoço e no jantar, respectivamente. Em relação às frutas, fontes de vitamina $C$, destacaram-se a laranja, a banana, o mamão e o maracujá, como as mais consumidas.

\section{DISCUSS Ã O}

Este estudo explora a relação entre os níveis de retinol sérico e indicadores bioquímicos do estado nutricional relativo ao ferro em uma população de crianças e adolescentes, da região Sudoeste da Bahia. Os resultados deste estudo indicam associação positiva e estatisticamente significante entre os níveis de retinol sérico e os níveis de hemoglobina $(p=0,007)$, ferro sérico $(p=0,010)$ e transferrina saturada (\%TS) $(p=0,027)$, mesmo quando estes resultados são ajustados pelas variáveis: demográficas, infecções 
parasitárias (S. mansoni e geohelmintos), consumo de ferro biodisponível e condições ambientais e domiciliares. Vários estudos têm indicado que o aumento dos níveis de vitamina A pode favorecer positivamente os níveis de hemoglobina ${ }^{9,10-23}$, de ferro sérico ${ }^{9-11,19}$, de transferrina saturada ${ }^{11,19}$, e a capacidade ligadora de ferro ${ }^{24}$ em diversos grupos etários de várias partes do mundo.

A interação positiva entre vitamina $\mathrm{A}$ e a absorção de ferro tem sido identificada em diversos estudos, entretanto, o mecanismo pelo qual esta interação ocorre ainda não está totalmente esclarecido. É possível que a vitamina $\mathrm{A}$ não interfira diretamente na absorção do ferro no trato intestinal, mas atue mobilizando as reservas de ferro, disponibilizando-as para a formação de hemoglobina. Outro possível mecanismo é explicado pelo papel estimulador da vitamina A na síntese de transferrina, aumentando o transporte de ferro do plasma para os tecidos ${ }^{25}$.

No presente estudo, $17,6 \%$ dos escolares encontravam-se anêmicos, identificados a partir dos critérios preconizados. A prevalência da anemia observada neste estudo pode ser explicada também pelo padrão alimentar dos sujeitos da investigação (Tabela 3), caracterizado pelo consumo predominante de feijão com farinha, acompanhado de baixa porção de alimentos fontes alimentares de ferro heme, a exemplo das carnes; além da pequena inserção no cardápio de frutas e verduras, alimentos favorecedores da absorção do ferro não heme, conforme identificaram Brito et al. ${ }^{12}$.

Assim, os resultados deste estudo corroboram aqueles que identificam que a deficiência de vitamina A se associa com o estabelecimento da anemia e podem ser amparados pelas conclusões de investigações, que têm indicado que suplementação com vitamina A exerce efeito positivo na elevação dos níveis hematológicos de ferro.

Ainda assim, devem ser destacadas que, embora as evidências sustentem o papel relevante dos determinantes nutricionais na determinação e evolução da anemia, postula-se o papel relevante dos fatores estruturais, representados pelas inadequadas condições socioeconômicas dos indivíduos na determinação da anemia. $\mathrm{Na}$ área de estudo, 50\% dos escolares vivem em precárias condições socioeconômicas e ambientais, 1/3 das famílias contam com menos de 1 salário-mínimo para a sobrevivência dos seus membros e, aproximadamente, 50\% dos chefes de família são analfabetos. As precárias condições socioeconômicas influenciam ainda a exposição às infecções parasitárias e limitam o acesso aos cuidados de saúde e ao saneamento básico. Esses mesmos fatores são reconhecidos como constrangedores do crescimento e da saúde de crianças e adolescentes, em especial no Nordeste brasileiro ${ }^{26}$.

Nesse sentido, são necessárias a definição de políticas e estratégias de intervenção no campo da saúde e nutrição voltadas para crianças em idade escolar e adolescentes, a exemplo das existentes para as crianças menores de 5 anos de idade, com vistas a erradicar deficiências nutricionais especificas e garantir o padrão de crescimento compatível com o potencial genético desses grupos populacionais. Não passa despercebida, também, a necessidade de definição de políticas no plano estrutural que garantam condições adequadas de saneamento e abastecimento de água tratada; ações factíveis e reconhecidas como promotoras de mudanças positivas permanentes nas condições de vida e saúde das populações.

\section{COLABORADORES}

R.C.R. SILVA participou da concepção, coleta de dados, análise e interpretação dos dados. A.M.O. ASSIS participou da concepção, coleta de dados, análise e interpretação dos dados. M.L. BARRETO participou da concepção, coleta de dados, análise e interpretação dos dados. M.L.P. SANTANA participou da coleta de dados. L.L. BRITO participou da coleta de dados e da interpretação dos resultados. M.G. REIS participou da coleta de dados. I.M. PARRAGA participou da concepção e da coleta de dados. R.E BLANTON participou da concepção, coleta de dados, análise e da interpretação dos dados.

\section{REFERÊ NCIAS}

1. Poveda E, Cuartas A, Guarin S, Forero Y, Villarreal E. Iron and vitamin A micronutrient status, risk 
factors for their deficiencies and anthropometric assessment in preschool child from Funza municipality, Colombia. Biomedica (Bogotá). 2007; 27(1):76-93

2. Mejia LA, Hodges RE, Rucker RB. Role of vitamin A in the absorption, retention and distribution of iron in the rat. J Nutr. 1979; 109(1):129-37.

3. Hodges RE, Rucker RB, Gardner RH. Vitamin A deficiency and abnormal metabolism of iron. Ann N Y Acad Sci. 1980; 355:58-61.

4. Mejia LA, Arroyave G. Biological interrelation between vitamin $A$ and iron. Arch Latinoam Nutr. 1982; 32(1):32-7.

5. Ameny MA, Raila J, Walzel E, Schweigert FJ. Effect of iron and/or vitamin A re-supplementation on vitamin $A$ and iron status of rats after a dietary deficiency of both components. J Trace Elem Med Biol. 2002; 16(3):175-8.

6. Panth M, Shatrugna V, Yasodhara P, Sivakumar B. Effect of vitamin A supplementation on haemoglobin and vitamin A levels during pregnancy. Br J Nutr. 1990; 64(2):351-8.

7. Suharno D, West CE, Muhilal, Logman MH, Waart FG, Karyadi D, et al. Cross-sectional study on the iron and vitamin A status of pregnant women in West Java, Indonesia. Am J Clin Nutr. 1992; 56(6):988-93.

8. Mejia LA, Chew F. Hematological effect of supplementing anemic children with vitamin $A$ alone and in combination with iron. Am J Clin Nutr. 1988; 48(3):595-600.

9. Bloem MW, Wedel M, Egger RJ, Speek AJ, Schrijver J, Saowakontha S, et al. Iron metabolism and vitamin A deficiency in children in northeast Thailand. Am J Clin Nutr. 1989; 50(2):332-8.

10. Bloem MW, Wedel M, van Agtmaal EJ, Speek AJ, Saowakontha S, Schreurs WH. Vitamin A intervention: short-term effects of a single, oral, massive dose on iron metabolism. Am J Clin Nutr. 1990; 51(1):76-9.

11. Ahmed F, Barua S, Mohiduzzaman M, Shaheen N, Bhuyan MA, Margetts BM, et al. Interactions between growth and nutrient status in schoolage children of urban Bangladesh. Am J Clin Nutr. 1993; 58(3):334-8.

12. Brito LL, Barreto ML, Silva RC, Assis AM, Reis MG, Parraga I, et al. Risk factors for iron-deficiency anemia in children and adolescents with intestinal helminthic infections. Rev Panam Salud Publica. 2003;14(6):422-31.

13. United Nations Children's Fund. Preventing iron deficiency in women and children: background and consensus on key technical issues and resources for advocacy, planning and implementing national programmes. New York: UNICEF; 1998.
14. Bieri JG, Tolliver TJ, Catignani GL. Simultaneous determination of alpha-tocopherol and retinol in plasma or red cells by high pressure liquid chromatography. Am J Clin Nutr. 1979; 32(10): 2143-9.

15. von Schenck $H$, Falkensson M, Lundberg B. Evaluation of "HemoCue," a new device for determining hemoglobin. Clin Chem. 1986; 32(3):526-9.

16. Pereira Jr DB. Utilization of the Kato-Katz-method (thick-smear technique) the diagnosis of Isospora (author's transl). Rev Bras Pesq Med Biol. 1979; 12(4-5):351-2.

17. Philippi ST, Szarfarc SC, Laterza CR. Virtual Nutri. Sistema de análise nutricional. Versão 1.0 for Windows. São Paulo: Universidade de São Paulo; 1996.

18. Monsen ER, Balintfy JL. Calculating dietary iron bioavailability: refinement and computerization. J Am Diet Assoc. 1982; 80(4):307-11.

19. Majia LA, Hodges RE, Arroyave G, Viteri F, Torun B. Vitamin A deficiency and anemia in Central American children. Am J Clin Nutr. 1977; 30(7): 1175-84.

20. Mohanram M, Kulkarni KA, Reddy V. Hematological studies in vitamin A deficient children. Int J Vitam Nutr Res. 1977; 47(4):389-93.

21. Li JH, Piao JH, Guo N, Yang LC. Study on the correlation of vitamin A and iron status in children. Wei Sheng Yan Jiu. 2006; 35(2):182-3.

22. Zimmermann MB, Biebinger $R$, Rohner $F$, Dib A, Zeder C, Hurrell RF, et al. Vitamin A supplementation in children with poor vitamin $A$ and iron status increases erythropoietin and hemoglobin concentrations without changing total body iron. Am J Clin Nutr. 2006; 84(3): 580-6.

23. Mwanri L, Worsley A, Ryan P, Masika J. Supplemental vitamin A improves anemia and growth in anemic school children in Tanzania. J Nutr. 2000; 130(11):2691-6.

24. Mejia LA, Arroyave G. The effect of vitamin A fortification of sugar on iron metabolism in preschool children in Guatemala. Am J Clin Nutr. 1982; 36(1):87-93.

25. Staab DB, Hodges RE, Metcalf WK, Smith JL. Relationship between vitamin $A$ and iron in the liver. J Nutr. 1984; 114(5):840-4.

26. Duarte EC, Schneider MC, Sousa R, Ramalho WM, Sardinha LM. Epidemiologia das desigualdades em saúde no Brasil: um estudo exploratório. Brasília: OPS; 2002.

Recebido em: 26/10/2006 Versão final reapresentada em: 18/9/2007 Aprovado em: 29/2/2008 